\title{
Effect of Water Adsorption on Cation-Surface Interaction Energy in the Na-Mordenite of 5.5 : 1 Si/Al Ratio
}

\author{
Sekou Diaby \\ Laboratoire de Chimie-Physique, Unité de Formation et de Recherche en Sciences des Structures de la Matière et Technologie (UFR- \\ SSMT), Université FHB de Cocody-Abidjan, 22 BP 582, Abidjan 22, Côte d'Ivoire
}

Correspondence should be addressed to Sekou Diaby; sekoudiaby@yahoo.fr

Received 2 October 2015; Accepted 18 January 2016

Academic Editor: Talhi Oualid

Copyright (C) 2016 Sekou Diaby. This is an open access article distributed under the Creative Commons Attribution License, which permits unrestricted use, distribution, and reproduction in any medium, provided the original work is properly cited.

\begin{abstract}
The mobility of the $\mathrm{Na}^{+}$cations localized at the inner surface of the studied mordenite zeolite depends on the material surface properties. In this work, we show that the activation energy, $\Delta E_{\text {hop }}$, relating to the $\mathrm{Na}^{+}$cation hopping displacement is associated to the surface potential and therefore can be used to get a better insight into the zeolite surface properties. Indeed, when molecules as water are adsorbed at the surface, they modify the surface potential energy and hence influence the value of $\Delta E_{\text {hop }}$. If the adsorbed molecules are polar they directly interact with the cations which become more mobile. The more the $\Delta E_{\text {hop }}$ value is, the less the amount of adsorbed water molecules is. Alterations of the $\Delta E_{\text {hop }}$ value with respect to the amount of adsorbed water molecules are interpreted using the Dubinin model which is based on simple adsorption principle.
\end{abstract}

\section{Introduction}

Zeolites are crystalline aluminosilicate minerals with highly interconnected latticeworks of nanopores. These materials are thus characterized by an important surface area which gives them very interesting properties to be utilized in adsorption and catalysis. There are hundreds of zeolites showing differences in the geometric shape of the nanopore and the chemical features of the nanopore surface. The latter is directly related to the zeolite $\mathrm{Si} / \mathrm{Al}$ ratio since the substitution of an $\mathrm{Al}$ atom for a $\mathrm{Si}$ one yields an excess of negative electrical charges which is compensated by cations localized at the nanopore surface. These cations are so-called extraframework (later abbreviated EFC) or compensation cations. The type and density of EFC at the surface play a crucial role in the surface properties. For instance, the presence of $\mathrm{H}^{+}$ or of some metallic cations is known to be the most relevant feature that controls the adsorption and catalysis efficiency of a given zeolite. Therefore, the accurate knowledge of the EFC localization at the surface as well as their nature and density is imperative for a better understanding of the zeolite surface properties and hence for a better control of their practical uses.
Owing to electrostatic interaction, the polarity of water molecules strongly favours their adsorption on or near the cations. Hydration and dehydration (which are important practical issues) of the zeolites are therefore much dependent on the EFC. Basically, it is thought that the solvation of the EFC by the adsorbed water molecules reduces the direct interaction between the EFC and the zeolite framework and consequently enhances the EFC mobility. The change in the EFC mobility with the amount of water molecules adsorbed into the zeolite can thus be seen as a significant outcome for characterizing the water adsorption process and the zeolite surface properties.

In this paper, we investigate the water adsorption into a mordenite zeolite with $5.5: 1 \mathrm{Si} / \mathrm{Al}$ ratio and its influence on the EFC $\mathrm{Na}^{+}$. The amount of adsorbed water is determined using thermogravimetric analysis (TGA). Besides, Dielectric Relaxation Spectroscopy (DRS) which is well known to be a very convenient tool to probe electrical charge displacement or dipole reorientation is used to measure the evolution of the EFC mobility as a function of the amount of adsorbed water molecules and of the temperature. The activation energy for the $\mathrm{Na}^{+}$mobility, $\Delta E_{\text {hop }}$, can be then determined from the Arrhenius treatment of the DRS data. The experimental 
modification of $\Delta E_{\text {hop }}$ with respect to the amount of adsorbed water molecules is finally interpreted using the Dubinin model which is based on simple adsorption principle.

The paper is constructed as follows. In Section 2.1, we recall some of the structural features of the mordenite zeolite studied here. In Section 2.2, the experimental methods used for measuring the amount of adsorbed water (TGA) and the EFC mobility (DRS) are briefly described. The Dubinin model is given in Section 2.3 whereas the analysis of the experimental results with this model is reported in Section 3.1. In Section 3.2, we discuss the data and their analysis. Conclusion is in Section 4.

\section{Materials and Methods}

2.1. The $\mathrm{Na}^{+}$Mordenite. The sodium mordenite is an aluminosilicate of the formula

$$
\left(\mathrm{Na}^{+}\right)_{x} \mathrm{Al}_{x} \mathrm{Si}_{48-x} \mathrm{O}_{96}, y \mathrm{H}_{2} \mathrm{O}
$$

Its structure consists of a linkage of tetrahedral compounds $\left(\mathrm{SiO}_{4}\right)^{4-}$ and tetrahedral compounds $\left(\mathrm{AlO}_{4}\right)^{5-}$, joined between them through their vertexes. Like the zeolite, the sodium mordenite is characterized by its structural unit otherwise called unit cell (u.c). It is comprised of tetrahedrons whose centres are occupied by silicon cations $\left(\mathrm{Si}^{4+}\right)$, or aluminium cations $\left(\mathrm{Al}^{3+}\right)$ whereas the oxygen atoms strongly attached to these centre cations occupy the vertexes of the tetrahedrons that link together in various ways. Each of the four tetrahedron oxygen atoms can exchange with the centre cation, an electron which causes a negative charge to arise when the concerned cation is trivalent as is $\mathrm{Al}^{3+}$. Then this charge is balanced by a $\mathrm{Na}^{+}$cation referred to as a compensation cation or EFC (for the alkaline group or alkaline earth group). The crystalline arrangement of the tetrahedral compounds, shaped like rings between them, is imparted to the mordenite zeolite porous properties. The compensation cations while being responsible for the catalysis do not form part of the open structure of the mineral, and under certain conditions one can replace them by other cations without altering the crystal structure. Thus, channels large enough to let the ions in and out appear throughout this crystal lattice. We showed a unit cell of mordenite with a diagram in Figure 1, and we indicated the different cationic sites I, II, III, IV, and VI denoted following Mortier's classification system for the crystallographic sites [1].

The pore size varies according to the nature of the additional cation, electrical neutrality being maintained. The compensation cations contribute a lot to many properties of the mordenite zeolites. They particularly attract polar molecules like those of water. So some mordenite zeolites hold, besides the alkaline ions and alkaline earth ions, water molecules in the ducts, and cavities located within the framework of the aluminosilicates. Thus, under the effect of heat energy, a Na-mordenite crystal is expelled from its framework, water molecules. However, the crystal does not become smaller. It conserves its initial measurements and shape. The places that occupied the water molecules remain unoccupied, making the dehydrated Na-mordenite

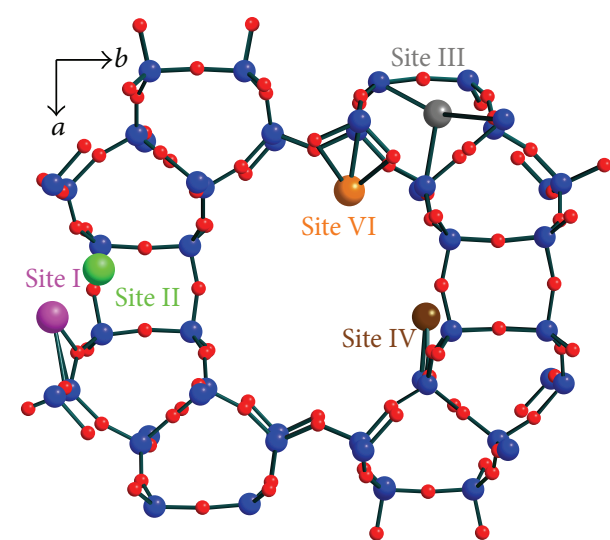

$$
\begin{aligned}
& \mathrm{Si} \\
& \mathrm{Al} \\
& \mathrm{O}
\end{aligned}
$$

FIgURE 1: Showing the cationic sites within a unit cell of $\mathrm{Na}$ mordenite [1].

into a desiccant or an adsorbent for the water vapour or for other gases. Numerous industrial methods involving heterogeneous catalytic reactions like oil refining generally employ zeolites. These reactions use elemental processes that take place at the interfacial area separating the solid catalyst from the fluid phases containing the reactants and products. So it is essential to have sufficient knowledge of reactive molecule fixation on the solid and then expect to master the successive steps in this heterogeneous catalysis.

\subsection{Experimental Methods}

2.2.1. Thermogravimetric Analysis (TGA) Measurements. The Na-mordenite sample placed in the capacitor as a dielectric is prepared in the following way: first, the zeolite is finely ground in an agate mortar and then it is compacted in the form of a disc, $15 \mathrm{~mm}$ thick and $1 \mathrm{~mm}$ in diameter, under a pressure of $10^{8} \mathrm{~Pa}$. Then, it is placed for 24 hours under an atmosphere saturated with water vapour, at temperature $T=298 \mathrm{~K}$ for the purpose of being completely hydrated. A series of measurements performed by using thermogravimetric analysis (TGA) allows the number of water molecules fastened per unit cell (u.c) to be determined for a series of treatment temperatures (TT). These measurements are carried out when inputting a known quantity of powdered zeolite fully hydrated following the steps earlier outlined, into an apparatus (SETARAM, Labsys ${ }^{\mathrm{TM}}$ ). The mordenite zeolite is submitted to heating, at the fixed rate of $4^{\circ} \mathrm{C}$ per hour, under a flow of perfectly dried argon up to the equilibrium characterized by the treatment temperature (TT). The apparatus measures uninterruptedly the mass losses due to the departure of water molecules and makes it possible to determine the remaining quantity of water, at equilibrium, in the sample, at each treatment temperature (TT) ranging from $100^{\circ} \mathrm{C}$ to $220^{\circ} \mathrm{C}$. As shown in Figure 2, we plotted the variations in the number $\left(N_{a}\right)$ of the adsorbed water 


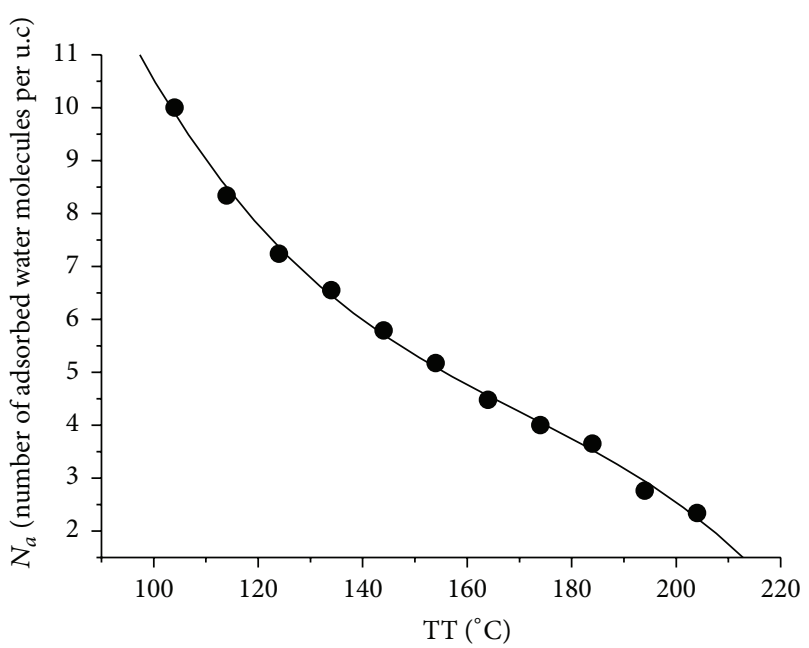

Figure 2: Number $\left(N_{a}\right)$ of adsorbed water molecules per unit cell (u.c) of Na-mordenite as a function of treatment temperature (TT) to which the zeolite was heated.

molecules per unit cell of Na-mordenite, as a function of the treatment temperature (TT), at which the zeolite is held.

\subsubsection{Study by Means of Dielectric Relaxation Spectroscopy (DRS)}

(1) Electric Polarization. Unlike the conductors that contain free charges moving around the medium, under the effect of an electric field, the dielectrics are substances whose electrons are strongly linked to their atom so that one can observe neither a courant due to the free electrons nor a courant induced by the displacements of ions. By applying an electric field, however, one observes in this poor electricity conductor, that is, the dielectric, tiny displacements of unlike charges in opposite directions, and this relative movement causes dipoles to appear; the dielectric is said to be polarized. Many different mechanisms can be responsible for the development of these electric dipoles, but the experiments show that an induced polarization arises in any dielectric submitted to an electric field. The microscopic laws that describe this polarization, in the case of the polar dielectrics, allow the relative permittivity to be defined as a complex quantity $\left(\varepsilon_{r}^{*}\right)$ :

$$
\varepsilon_{r}^{*}=\varepsilon_{r}^{\prime}-j \times \varepsilon_{r}^{\prime \prime}
$$

Then one shows [2-6] that, under the effect of an alternating electric field of angular frequency $\omega$, this relative permittivity varies according to the relaxation time $(\tau)$ that characterizes the movement that brings dipoles into alignment with this field:

$$
\varepsilon_{r}^{*}=\varepsilon_{r, \infty}+\frac{\varepsilon_{r, 0}-\varepsilon_{r, \infty}}{1+j \times \omega \times \tau} .
$$

By using (2) to separate the imaginary and real parts of (3), one gets

$$
\begin{aligned}
& \varepsilon_{r}^{\prime}=\varepsilon_{r, \infty}+\frac{\varepsilon_{r, 0}-\varepsilon_{r, \infty}}{1+(\omega \times \tau)^{2}}, \\
& \varepsilon_{r}^{\prime \prime}=\frac{\left(\varepsilon_{r, 0}-\varepsilon_{r, \infty}\right) \times(\omega \times \tau)}{1+(\omega \times \tau)^{2}} .
\end{aligned}
$$

It is noted, regarding these equations, that the curves schematizing the value of $\varepsilon_{r}^{\prime \prime}$ as a function of $\omega$, for a given value of $\tau$, pass through a peak when $\omega=1 / \tau$. In actual practice, it is noticeable that the peaks of these curves move towards the high values of $\omega$ when the temperature $(T)$ of the sample rises.

(2) Jumping Mechanism. This last remark inspired us to bring in a microscopic modelling for relating the relaxation time $(\tau)$ to the temperature $(T)$. In this model, each of the component particles of the studied sample is placed at the minimum of a potential well when it is in its equilibrium position. It can climb out of there only when the thermal motion $[7,8]$ enables it to reach an energy level high enough to make it possible to cross over a potential barrier characteristic of its structural environment. If one imagines its state when the system is held at $T=0 \mathrm{~K}$, each charge carrier is trapped into a site whose geometry solely determines its frequency of oscillations $\left(\tau_{0}^{-1}\right)$ in this fundamental state $[9,10]$. The thermal motion provides additional energy for it, which is excited to a state of higher energy, and increases the likelihood of it crossing over the potential barrier $\left(\Delta E_{\text {hop }}\right)$ separating the two neighbouring positions characterized by the same fundamental energy. This operation enables it to move between two neighbouring equilibrium positions. This description of an elemental jumping also indicates that the value of the barrier $\left(\Delta E_{\text {hop }}\right)$ gives a measure of the energy associated with the pseudo dissociation reaction of the matching pair of charge carrier-site. Then, one can use Maxwell-Boltzmann's distribution law to calculate the number of charge carriers likely to climb out of this potential well, under the effect of the thermal motion, which allows us to express the relaxation time of the sodium cations as

$$
\tau=\tau_{0} \times \exp \left[\frac{\Delta E_{\mathrm{hop}}}{k \times T}\right],
$$

where $k=1.38 \times 10^{-23} \mathrm{~J} \cdot \mathrm{K}^{-1}$ is the Boltzmann constant. This equation can be written in the following form:

$$
\log \left(\frac{1}{\tau}\right)=\log \left(\frac{1}{\tau_{0}}\right)-\frac{\Delta E_{\text {hop }}}{k \times T}
$$

The dipoles that came into being in the phenomenon of polarization within the studied Na-mordenite of $5.5: 1 \mathrm{Si} / \mathrm{Al}$ ratio are oriented from the side of the rigid network of the aluminium silicate where the negative pole materializes, towards the side of the compensation cations $\left(\mathrm{Na}^{+}\right)$, located in the sites I, IV, and VI [11-13], that is, in turn the positive pole. The calculations show that, in the system, $\tau_{0}$ varies 
slightly from one site to another and always remains very close to a value such that $\log \left(1 / \tau_{0}\right)=30.61$.

(3) Measuring Method. The Na-mordenite sample compacted under a pressure of $10^{8} \mathrm{~Pa}$, in the form of a disc, is placed between the conducting surfaces separated by a distance $(d)$ of a plane capacitor whose vacuum capacitance is denoted by $C_{0}$. When one applies across the capacitor a sinusoidal tension $U(\omega)$, with an angular frequency $(\omega)$, an electric polarization $(\vec{p})$ occurs, with a phase difference relative to the electric field $\vec{E}$. Under these conditions, the electric current $(I(\omega))$, flowing through the capacitor, is expressed as a complex quantity (7) and with the use of (2) can be rearranged as a sum of a real part and an imaginary one (8). Consider the following:

$$
\begin{aligned}
& I(\omega)=U(\omega) \times\left[j \times \omega \times \varepsilon_{r}^{*} \times C_{0}\right] \\
& I(\omega)=U(\omega) \times\left[\left(\omega \times \varepsilon_{r}^{\prime \prime} \times C_{0}\right)+j \times\left(\omega \times \varepsilon_{r}^{\prime} \times C_{0}\right)\right] .
\end{aligned}
$$

The capacitor filled with Na-mordenite can also be described by an equivalent circuit, formed of a parallel connection of a resistance $(R)$ with a capacitor (with capacitance $C$ ). The admittance of such circuit is written as $[(1 / R)+j \times C \times \omega]$ and allows calculating the electric current $I(\omega)$ which passes in this capacitor when it is subjected to a tension $U(\omega)$ :

$$
I(\omega)=U(\omega) \times\left[\frac{1}{R}+j \times C \times \omega\right] .
$$

The comparison of (8) and (9) leads to

$$
\begin{aligned}
\varepsilon_{r}^{\prime \prime} & =\frac{1}{R \times C_{0} \times \omega}, \\
\varepsilon_{r}^{\prime} & =\frac{C}{C_{0}} .
\end{aligned}
$$

In actual practice, by the aid of an impedance-meter (NOVOCONTROL), we determined the characteristics of a capacitor with the Na-mordenite as dielectric by measuring its capacitance $(C)$ and its conductance $(G=1 / R)$, when it is submitted to a $0.5 \mathrm{~V}$ alternating electric field, whose angular frequency ranges from $6.28 \times 10^{-3} \mathrm{rad} \times \mathrm{s}^{-1}$ to $6.28 \times 10^{+7} \mathrm{rad} \times \mathrm{s}^{-1}$.

\subsection{Gas Adsorption in Solids and the Dubinin Model}

2.3.1. Reminder of Some Modelling of Gas Adsorption on Some Solids. As far back as 1916, Langmuir $[14,15]$ went through a model likely to account for a fully gaseous monomolecular layer adsorption on a solid surface, by assuming that the molecules making up this layer do not interact between them. This model was designed for interpreting the formation of the monolayer. This assumes that the evaporation rate $\left(v_{e}\right)$ is proportional to the fraction $\left(\theta=N_{a} / N_{0}\right)$ of the surface area covered at any given time by the adsorbed molecules, $N_{a}$ being the number of adsorbing molecules or adsorbates fastened on the surface and $N_{0}$ the total number of the adsorption sites. The condensation rate $\left(v_{c}\right)$ of the molecules on the surface is proportional to the surface not yet covered $(1-\theta)$ and the pressure $P$ on the gas. At equilibrium, the condensation rate $\left(v_{c}=k_{c} \times P \times(1-\theta)\right)$ of the molecules on the surface is equal to the evaporation rate $\left(v_{e}=k_{e} \times \theta\right)$ of the molecules from the surface, given with

$$
k_{c} \times P \times(1-\theta)=k_{e} \times \theta .
$$

One can write

$$
\frac{P}{N_{a}}=\frac{P}{N_{0}}+\frac{1}{K(T) \times N_{0}} .
$$

Denoting by $K(T)=k_{c} / k_{e}$, equilibrium is constant. Thus, when one knows the surface covered by an adsorbing molecule, one can deduce from the Langmuir isotherms, corresponding to (12), the specific surface area of the adsorbent.

In 1938, Brunauer et al. [16] proposed a new model of the adsorption phenomenon, taking into account the formation of several molecule layers adsorbed and gas-gas interaction on the one hand and gas-solid ones on the other hand. They have got the following equation called BET equation, which enables a convenient representation of the adsorption isotherms to be obtained:

$$
\frac{P}{\left(P-P_{0}\right) \times N_{a}}=\frac{1}{c \times N_{0}}+\left[\frac{c-1}{c \times N_{0}} \times \frac{P}{P_{0}}\right],
$$

where

$$
c=\exp \left[\frac{E_{1}-E_{\ell}}{R \times T}\right] .
$$

The quantity $c$ provides information on the affinity of the adsorbed molecules with the solid. The energy $E_{1}$ constitutes an estimate of the heat of adsorption by the prime layer, and energy $E_{\ell}$ is a measure of the adsorbate condensing heat, $R$ is the universal gas constant, and $T$ is the isotherm temperature. The graphic representation of (13), for the values of the ratio $P / P_{0}$, such that $0.05 \leq P / P_{0} \leq 0.3$, allows on the one hand the quantity $c$ and on the other hand the specific area of the adsorbent to be determined.

In 1940, Dubinin, by analyzing a whole series of experimental results, contributed interestingly to describing the gaseous molecule adsorption phenomenon on microporous solids. He showed clearly a correlation by relating the amount of adsorbed gas to the energy involved in the molecule fixation mechanism on the surface. In cooperation with many other authors [17-22], he compared this energy to $-\Delta G$, $\Delta G$ being Gibbs free energy change of the transformation reaction from the adsorbed phase to the gaseous one, which is described by the following chemical equation:

$$
\begin{aligned}
& \text { Molecule }_{\text {Adsorbed, at } T \text { and } P_{0}} \\
& \qquad \text { Molecule }_{\text {in gas phase, at } T \text { and } P}
\end{aligned}
$$

Applying the laws of thermodynamics on equilibrium states, then one obtains

$$
\begin{aligned}
\Delta G+R \times T \times \operatorname{Ln}\left[\frac{P}{P_{0}}\right] & =0 \\
\text { hence }-\Delta G & =R \times T \times \operatorname{Ln}\left[\frac{P}{P_{0}}\right] .
\end{aligned}
$$


For a given system of adsorbate-adsorbent, Dubinin showed that the graphic representation of the adsorption rate $(\theta=$ $\left.N_{a} / N_{0}\right)$, as a function of $P / P_{0}$, yields only one curve, referred to as a characteristic. In the case of a given solid, the characteristic curves of different adsorbates can be fitted upon each other by means of using a specific parameter $(\beta)$ called affinity whose value is dependent upon the nature of the adsorbate. The value of an energy $E_{0}$ characterizing the structure of the solid and related to the width $L$ of the pores existing on its surface is a measure of the adsorbent influence that is given with

$$
L=\frac{10.8}{\left(E_{0}-11.4\right)} .
$$

In 1947, Dubinin et al. [23, 24] put forward an equation allowing the description of the organic molecule adsorption on the activated carbons. Then, in 1971, Dubinin and Astakhov [25] expanded it and made it applicable to the other solids including the zeolite by adding an exponent $\eta$ that characterizes the solid's heterogeneousness given with

$$
\begin{aligned}
N_{a} & =N_{0} \times \exp \left[-\left[\frac{(-\Delta G)}{\beta \times E_{0}}\right]^{\eta}\right] \\
& =N_{0} \times \exp \left(-B \times(-\Delta G)^{\eta}\right) .
\end{aligned}
$$

We must repeat that $E_{0}$ and $B$ are characteristic quantities of the studied solid.

\section{Measurement Method and Interpretation}

\subsection{Experimental Data and Analysis}

3.1.1. Utilization of the Polarization Measurements. We reproduced the same hydration conditions and then placed a $\mathrm{Na}$-mordenite sample, held for three hours at a treatment temperature (TT) at equilibrium, in a measuring cell of an impedance-meter (NOVOCONTROL). Then we observed the variations in the imaginary part of the dielectric permittivity $\left(\varepsilon_{r}^{\prime \prime}\right)$ in relation to the angular frequency $(\omega)$ of the applied electric field $(\vec{E})$ and to the temperature $(T)$ imposed on the sample. First of all, we ensure that the number of water molecules fastened on the sample does not increase by means of an external action whereby it is kept under an atmosphere in equilibrium with its hydration rate and that with the use of a device operating with a flow of perfectly dry argon. Thus we know that, under these conditions, a process wherein water moves can partake only if the sample undergoes an increase in temperature. Then, we imposed on it a temperature series, each of which was inferior to TT-10, for determining the activation energy of the polarization phenomenon. Complying with these requirements, we measured the imaginary part of the permittivity $\left(\varepsilon_{r}^{\prime \prime}\right)$, according to the angular frequency $(\omega)$ of the applied electric field. As shown in Figure 3, we obtained a series of curves by drawing the variations in $\varepsilon_{r}^{\prime \prime}$ as a function of $\log (\omega / 2 \pi)$, at $\mathrm{TT}=373 \mathrm{~K}$ and $T$ varying from $323 \mathrm{~K}$ to $263 \mathrm{~K}$.

Referring to the curves, we $\operatorname{localized} \log \left(\omega_{m} / 2 \pi\right)$ corresponding to the abscissa of the maximum of $\varepsilon_{r}^{\prime \prime}$. Based

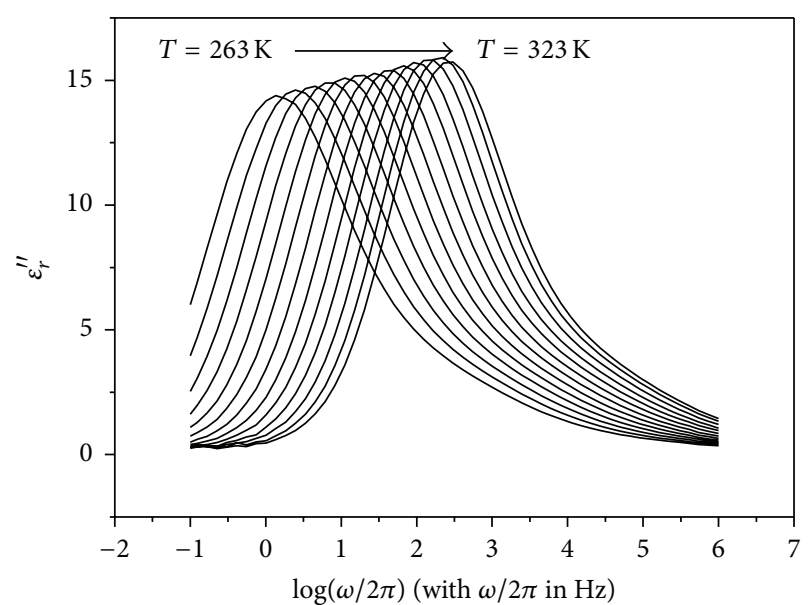

FIgURE 3: Loss factor $\varepsilon_{r}^{\prime \prime}$ in Na-mordenite of 5.5:1 Si/Al ratio (previously hydrated at the treatment temperature (TT)), as a function of $\log (\omega / 2 \pi)$ at each temperature $T$ such that $T<\mathrm{TT}-$ $10 \mathrm{~K}$.

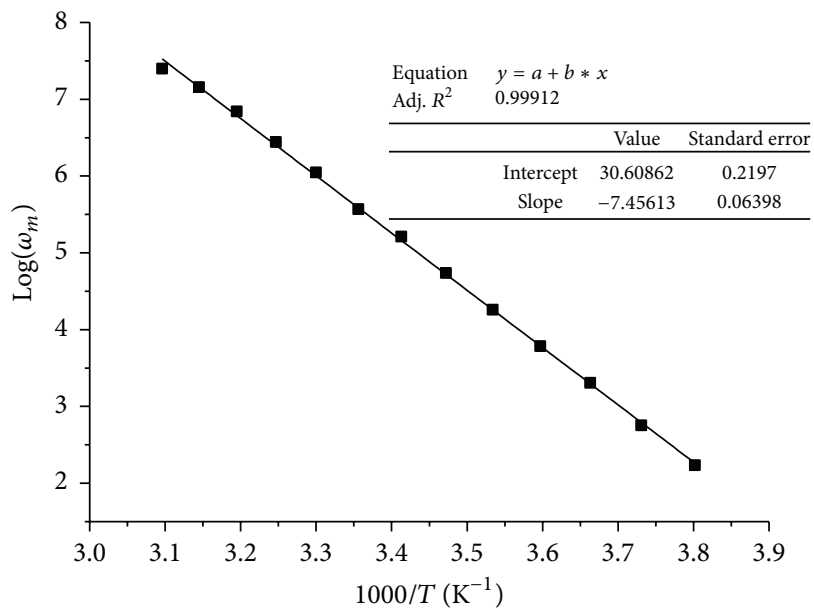

Figure 4: Using a Na-mordenite sample of 5.5:1 Si/Al ratio previously hydrated at the treatment temperature $\mathrm{TT}=373 \mathrm{~K}$, to ascertain experimentally the relationship $\log \left(\omega_{m}\right)=\log \left(1 / \tau_{0}\right)-$ $\Delta E_{\text {hop }} /(k \times T)$.

on (3), we know that this point ( $\varepsilon_{r}^{\prime \prime}$ peak maximum) is characterized by the relationship $\omega_{m} \times \tau=1$, whereof one derives straightforwardly $\tau=1 / \omega_{m}$, for each measuring temperature $T$. Then, one plots the values of $\log (1 / \tau)=$ $\log \left(\omega_{m}\right)$ thus obtained as a function of $1000 / T$, where $T$ is the temperature that was maintained constant throughout the determination of the curve: $\varepsilon_{r}^{\prime \prime}=f(\log (\omega / 2 \pi))$.

Then, one makes sure that the relationship (5) can be used while drawing the representative curve of $\log (1 / \tau)=$ $f(1000 / T)$, and checking, as illustrated in Figure 4, that one actually obtains a straight line whose slope allows $\Delta E_{\text {hop }}$ to be determined from (6) [26-28].

The value of the activation energy $\left(\Delta E_{\text {hop }}\right)$ calculated from the slope of the straight line drawn, as shown in Figure 4, is $\Delta E_{\text {hop }}=0.64 \mathrm{eV}$ for $\mathrm{TT}=373 \mathrm{~K}$. Thereafter we repeated the method that has just been described for 


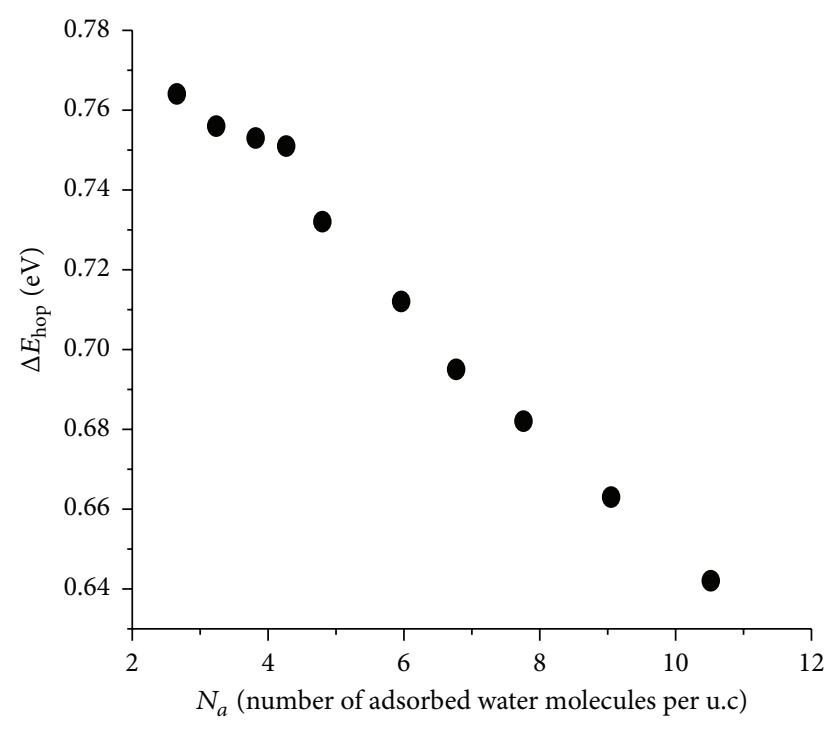

Figure 5: Change in the jumping energy $\left(\Delta E_{\text {hop }}\right)$ in a Na-mordenite of $5.5: 1 \mathrm{Si} / \mathrm{Al}$ ratio as a function of the number $\left(N_{a}\right)$ of adsorbed water molecules per unit cell (u.c).

determining the variations in the value of $\Delta E_{\text {hop, when }}$ the Na-mordenite sample of 5.5:1 Si/Al ratio is submitted to the treatment temperatures (TT) ranging from $333 \mathrm{~K}$ to $467 \mathrm{~K}$. The output of the measurements by differential thermal analysis (DTA) we previously performed is given with Figure 5.

Thus we showed that each treatment temperature of the $\mathrm{Na}$-mordenite of $5.5: 1 \mathrm{Si} / \mathrm{Al}$ ratio corresponds to an amount of adsorbed water molecules per unit cell that we already denoted by $N_{a}$. Figure 5 illustrates the graph of the value of $\Delta E_{\text {hop }}$ as a function of the number $\left(N_{a}\right)$ of adsorbed water molecules per unit cell of Na-mordenite of $5.5: 1 \mathrm{Si} / \mathrm{Al}$ ratio.

3.2. Discussion. The adsorbed water molecules fastened on the Na-mordenite are in equilibrium with those in vapour phase, in contact with the solid. The laws obeyed by the mechanism of this equilibrium can be studied when regarding it as a pseudo reaction given with

$$
\begin{aligned}
& \text { Molecule }_{\text {Adsorbed at } T \text { and } P_{0}} \\
& \Longleftrightarrow \text { Molecule }_{\text {in gas phase at } T \text { and } P}
\end{aligned}
$$

The laws of thermodynamics of the equilibrium states enable one to write

$$
-\Delta G=R \times T \times \log \left[\frac{P}{P_{0}}\right] .
$$

Many authors including Dubinin et al. [29-35] assimilated the energy which sustains the mechanism fixing the molecules adsorbed on the surface to the opposite of Gibbs free energy change $(-\Delta G)$ of the pseudo reaction. To interpret the observed behaviour, it is necessary to provide an estimate of the interaction energy between the adsorbed water molecules and the exchanged cations on the solid surface.
3.2.1. Making Use of a Lennard-Jones Potential to Describe the Interaction between a Compensation Cation and the Adsorbed Water Molecules. We assume that each interaction energy involved in the adsorption phenomena can be described by a Lennard-Jones potential [36-38]. By disregarding the term of repulsion whose influence is negligible in an adsorption process, we can write the relationship already suggested by Hasley, Hill, and Frenkel [39-41]:

$$
u=\sum_{j}\left[\frac{\kappa}{r_{j}}\right]^{n}
$$

where $u$ is the interaction energy between an adsorbed water molecule and the surface, $r_{j}$ is the distance between the adsorbed molecule and an atom on the surface that interacts with it, $\kappa$ is a constant, and $n$ is an empirical exponent.

Then one considers the stage of the adsorption process where the number of the adsorbed water molecules is lower than that which makes it possible for the monolayer to be formed, that is to say less than 10 adsorbed water molecules per unit cell of Na-mordenite. Previous works have shown that these water molecules are distributed on the solid surface to form a latticework [42-44]. Denoting by $r_{w w}$ the distance between two water molecules, by $r_{k w}$ the distance between a sodium cation and its closest neighbouring water molecules, by $N_{a}$ the number of adsorbed water molecules per unit cell of Na-mordenite, and by $A_{\sigma}$ the solid's total surface accessible to the water molecules, one can write the two following equations:

$$
\begin{aligned}
& A_{\sigma}=\alpha \times N_{a} \times r_{w w}^{2}, \\
& r_{k w}=\beta \times r_{w w},
\end{aligned}
$$

where $\alpha$ and $\beta$ are parameters that describe the lattice geometry. By combining (22) and (23), one derives

$$
r_{k w}=\beta \times \sqrt{\frac{A_{\sigma}}{\alpha \times N_{a}}} .
$$

Substituting the values of $r_{k w}$, obtained from (24), into (21), one can sum up the energies of a cation interaction with the adsorbed water molecules which are its closest neighbours. Thus one obtains an estimate of the variation in the energy $\left(\Delta E_{\mathrm{hop}}\right)$ caused by the first adsorbed water molecules, implying that $N_{a} \leq 10$. We incorporated the expression of $r_{k w}$ (24) into (21), and we obtained after due calculations the following expression of the variations in the jumping energy $\left(\Delta E_{\text {hop }}\right)$ under the effect of the $N_{a}$ water molecule adsorption:

$$
\Delta E_{\text {hop }}\left(N_{a}\right)=\Delta E_{\text {hop }}^{0}-N_{a} \times\left[\frac{\kappa \times \sqrt{\alpha \times N_{0}}}{\beta \times \sqrt{A_{\sigma}}}\right]^{n} .
$$

For greater convenience, we rearranged this equation as

$$
\Delta E_{\text {hop }}\left(N_{a}\right)=\Delta E_{\text {hop }}^{0}-B \times N_{a} \text {. }
$$

In expression (26), $\Delta E_{\text {hop }}^{0}$ stands for the cation extraction energy from its site under the conditions where the $\mathrm{Na}$ mordenite sample is fully dehydrated and $B$ is a coefficient 


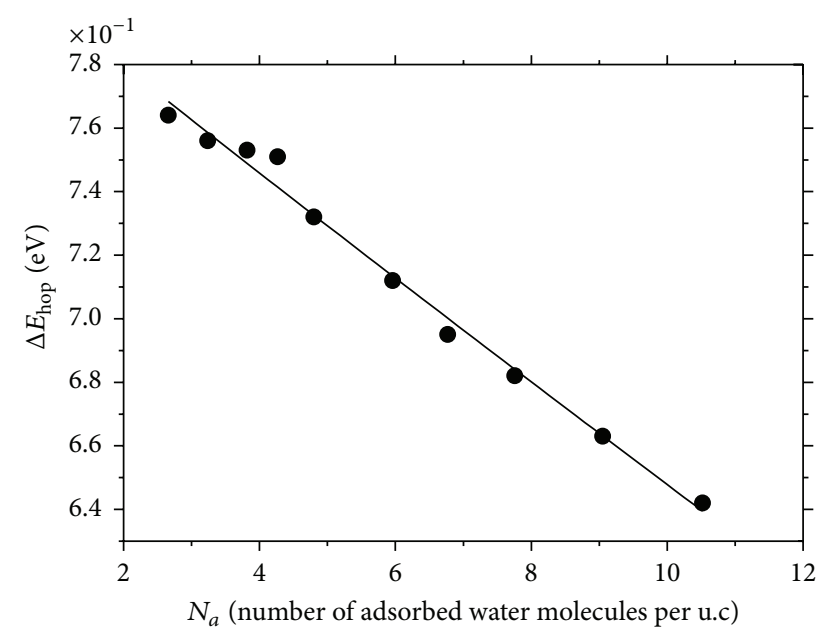

Figure 6: Fit between the curve schematizing the equation $\Delta E_{\text {hop }}\left(N_{a}\right)=\Delta E_{\text {hop }}^{0}-B \times N_{a}$ and the plot of the experimental values of $\Delta E_{\text {hop }}\left(N_{a}\right)$ as a function of the number $\left(N_{a}\right)$ of adsorbed water molecules per unit cell (u.c) of a Na-mordenite of $5.5: 1 \mathrm{Si} / \mathrm{Al}$ ratio.

of variation. Then we used (26), and try to find out the change in the value of $\Delta E_{\text {hop }}$ according to the number $\left(N_{a}\right)$ of adsorbed water molecules per unit cell of Na-mordenite of $5.5: 1 \mathrm{Si} / \mathrm{Al}$ ratio. The result is shown in Figure 6 whereon one sees that (26) allows the observed adsorption behaviour to be interpreted properly.

This well fit between the curves enables us to determine the following values:

$$
\begin{aligned}
\Delta E_{\text {hop }}^{0} & =0.81 \mathrm{eV}, \\
B & =0.0164 \mathrm{eV} \cdot \text { molécule }^{-1} .
\end{aligned}
$$

3.2.2. Making Use of Dubinin, Raduskevich, and Kaganer's Empirical Approach to Describe the Interaction between a Compensation Cation and the Adsorbed Water Molecules. The second aspect of the interpretation of the observed behaviours, based on the experimentally determined values of $\Delta E_{\text {hop }}\left(N_{a}\right)$ we plotted as a function of the number $\left(N_{a}\right)$ of the adsorbed water molecules per unit cell of Na-mordenite of $5.5: 1 \mathrm{Si} / \mathrm{Al}$ ratio, involves using the empirical approach suggested by Dubinin, Raduskevich, and Kaganer [29-32]. Indeed, these authors proposed an equation relating the ratio $\left(N_{a} / N_{0}\right)$ and the energy that is involved in the process of the molecule fixation on the surface. The equation is written as follows:

$$
\log \left[\frac{N_{a}}{N_{0}}\right]=-D_{\text {Dub }} \times u^{2}
$$

Assume that

$$
u=R \times T \times \log \left[\frac{P}{P_{0}}\right] .
$$

By using reasoning similar to that we developed in the previous section, we derive the following equation aiming at recovering the change in the value of $\Delta E_{\text {hop }}$ as a function of

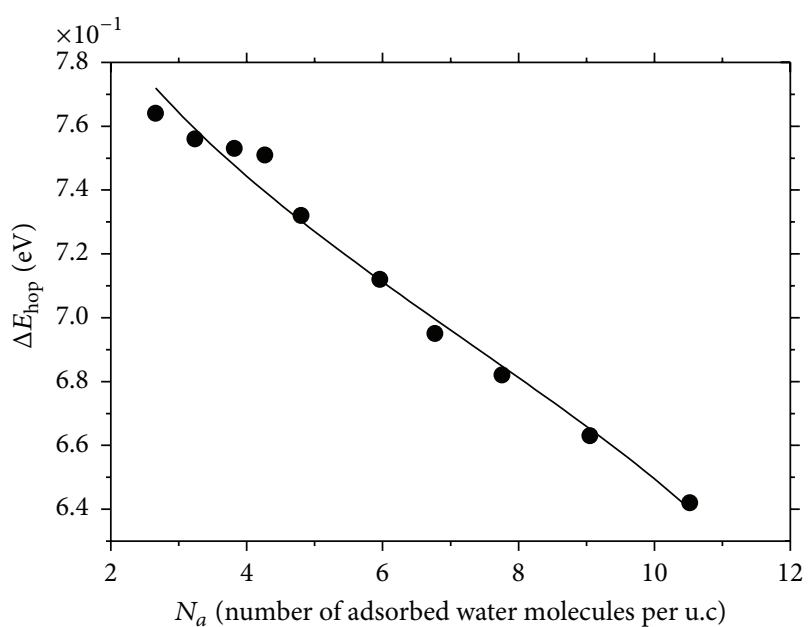

Figure 7: Comparison between the experimental plot of the variations in $\Delta E_{\mathrm{hop}}\left(N_{a}\right)$ and the curve schematizing the equation $\Delta E_{\text {hop }}\left(N_{a}\right)=\Delta E_{\text {hop }}^{T}+\sqrt{\left(\left[\log \left[N_{0} / N_{a}\right]\right) / D_{\text {Dub }}\right]}$, elicited from the empirical approach of Dubinin.

the number $\left(N_{a}\right)$ of water molecules adsorbed per unit cell of $\mathrm{Na}$-mordenite of $5.5: 1 \mathrm{Si} / \mathrm{Al}$ ratio:

$$
\Delta E_{\text {hop }}\left(N_{a}\right)=\Delta E_{\text {hop }}^{T}+\sqrt{\left[\frac{\log \left[N_{0} / N_{a}\right]}{D_{\text {Dub }}}\right]} .
$$

In that equation, $\Delta E_{\text {hop }}^{T}$ denotes the extraction energy of the compensation sodium cation from its site, under the conditions where the Na-mordenite surface is coated with a water molecule monolayer. It is shown in Figure 7 that the plot of the values of $\Delta E_{\text {hop }}\left(N_{a}\right)$ experimentally determined follows closely the shape of the curve schematizing $\Delta E_{\text {hop }}\left(N_{a}\right)=\Delta E_{\text {hop }}^{T}+\sqrt{\left[\log \left[N_{0} / N_{a}\right] / D_{\text {Dub }}\right]}$. Making that curve fit suitably upon the experimental plot then provides the following parameters:

$$
\begin{aligned}
\Delta E_{\text {hop }}^{T} & =0.58 \mathrm{eV} \\
D_{\text {Dub }} & =41.3223 \mathrm{eV}^{-2}
\end{aligned}
$$

and $N_{0}=12.17$ water molecules per unit cell of $\mathrm{Na}$-mordenite.

\section{Conclusion}

In this work, we carried out dielectric relaxation measurements to investigate experimentally the change in the jumping energy of the sodium cations in a Na-mordenite of $5.5: 1 \mathrm{Si} / \mathrm{Al}$ ratio, according to the degree of advancement of the water molecule adsorption. We also suggested two types of interpretations to account for the behaviours we observed:

(1) The first one issuing from the theory of Frenkel, Halsey, and Hill makes it possible to give an exact representation of the results we got experimentally. It allows determining the extraction energy of the sodium cations in a dehydrated zeolite. 
(2) The second one, based on the empirical approach suggested by Dubinin, Raduskevich, and Kaganer, also leads to a very satisfactory representation of the experimental results. An adequate handling of them provides an estimate of the extraction energy of the sodium cations in a zeolite when its surface is coated with a monolayer of adsorbing molecules and also an estimate of the number of the molecules that form the monolayer when the concerned molecules are those of water.

This paper suggests a new experimental approach that gives original information on elemental processes involved in the adsorption phenomenon enabling one to account quantitatively and in detail for the interactions between the solid surface, the compensating cations, and the molecules adsorbed onto the aluminosilicates characterized by the presence of pores wherein the exchangeable cations are trapped.

\section{Conflict of Interests}

The author declares that there is no conflict of interests regarding the publication of this paper.

\section{Acknowledgments}

The author would like very much to thank Professor J. C. Giuntini (Honorary Professor at University of Montpellier 2, France) and Professor F. Henn (Professor at University of Montpellier 2, France) for the Dielectric Relaxation Spectroscopy (DRS) facility and for their support.

\section{References}

[1] W. J. Mortier, Compilation of Extraframework Sites in Zeolites, Butterworth, Guildford, UK, 1982.

[2] E. Durand, Electrostatique I: Les Distributions, Masson, Paris, France, 1964.

[3] P. Baudoux, Précis D'électricité Fondamentale, Presses Universitaires de Bruxelles, Eyrolles, Paris, France, 1970.

[4] P. Debye, "Hochfrequenzverluste und molekulstruktur," Physikalische Zeitschrift, vol. 35, p. 101, 1934.

[5] K. S. Cole and R. H. Cole, "Dispersion and absorption in dielectrics I. Alternating current characteristics," The Journal of Chemical Physics, vol. 9, no. 4, pp. 341-351, 1941.

[6] C. J. F. Bottcher, Theory of Electrical Polarisation, Elsevier, Amsterdam, The Netherlands, 1973.

[7] I. Prigogine and V. Mathot, "Application of the cell method to the statistical thermodynamics of solutions," The Journal of Chemical Physics, vol. 20, no. 1, pp. 49-57, 1952.

[8] I. Prigogine and L. Saraga, "Sur la tension superficielle et le modèle cellulaire de l'état liquide," The Journal of Chemical Physics, vol. 195, pp. 399-407, 1952.

[9] R. H. Fowler and E. A. Guggenheim, Statistical Thermodynamics, Cambridge University Press, Cambridge, UK, 1965.

[10] F. Buet, J. C. Giuntini, F. Henn, and J. V. Zanchetta, "Microscopic considerations of the calculation of the polarization conductivity in ionically conducting glasses," Philosophical Magazine Part $B$, vol. 66, no. 1, pp. 77-88, 1992.
[11] W. H. Meir and D. H. Olson, "Atlas of zeolite structure type," Zeolites, vol. 17, p. 152, 1996.

[12] J. L. Schlenker, J. J. Pluth, and J. V. Smith, "Positions of cations and molecules in zeolites with the mordenite-type framework: VIII Dehydrated sodium-exchanged mordenite," Materials Research Bulletin, vol. 14, no. 6, pp. 751-758, 1979.

[13] P. F. Barbara, T. J. Meyer, and M. A. Ratner, "Contemporary issues in electron transfer research," The Journal of Physical Chemistry, vol. 100, no. 31, pp. 13148-13168, 1996.

[14] I. Langmuir, "The constitution and fundamental properties of solids and liquids. Part I. Solids," The Journal of the American Chemical Society, vol. 38, no. 2, pp. 2221-2295, 1916.

[15] I. Langmuir, "The adsorption of gases on plane surfaces of glass, mica and platinum," Journal of the American Chemical Society, vol. 40, no. 9, pp. 1361-1403, 1918.

[16] S. Brunauer, P. H. Emmett, and E. Teller, "Adsorption of gases in multimolecular layers," Journal of the American Chemical Society, vol. 60, no. 2, pp. 309-319, 1938.

[17] M. Polanyi, "On adsorption from the standpoint of the third law of thermodynamics," Verhandlungen der Deutschen Physikalischen Gesellschaft, vol. 16, pp. 1012-1016, 1914.

[18] M. Polanyi, "Adsorption of gases by a solid non-volatile adsorbent," Verhandlungen der Deutschen Physikalischen Gesellshaft, vol. 18, pp. 55-80, 1916.

[19] M. Polanyi, "On adsorption and the origin of adsorption forces," Zeitschrift für Elektrochemie, vol. 26, pp. 370-374, 1920.

[20] M. Polanyi, "Adsorption from solutions of substances of limited solubility," Zeitschrift für Physik, vol. 2, pp. 111-116, 1920.

[21] L. Berenyi, "Proof of Polanyi's theory of adsorption," Zeitschrift für Physikalische Chemie, vol. 94, pp. 628-662, 1920.

[22] L. Berenyi, "Modern adsorption calculations," Zeitschrift für Physikalische Chemie, vol. 105, pp. 55-72, 1923.

[23] M. M. Dubinin, E. D. Zaverina, and L. V. Radushkevich, "Sorption and structure of active carbons. I. Adsorption of organic vapors," Zhurnal Fizicheskoi Khimii, vol. 21, pp. 1351$1362,1947$.

[24] M. M. Dubinin, "Fundamentals of the theory of adsorption in micropores of carbon adsorbents: characteristics of their adsorption properties and microporous structures," Carbon, vol. 27, no. 3, pp. 457-467, 1989.

[25] M. M. Dubinin and V. A. Astakhov, "Development of the concepts of volume filling of micropores in the adsorption of gases and vapors by microporous adsorbents," Izvestiya Akademii Nauk SSSR, vol. 11, p. 5, 1971.

[26] S. Devautour, J. Vanderschueren, J. C. Giuntini, F. Henn, J. V. Zanchetta, and J. L. Ginoux, "Na ${ }^{+} /$mordenite interaction energy by thermally stimulated depolarization current," The Journal of Physical Chemistry B, vol. 102, no. 19, pp. 3749-3753, 1998.

[27] J. C. Giuntini, G. Maurin, S. Devautour, F. Henn, and J. V. Zanchetta, "Experimental evidence of polarization effects on exchangeable cations trapped in zeolites," Journal of Chemical Physics, vol. 113, no. 11, pp. 4498-4500, 2000.

[28] J. C. Giuntini, V. Mouton, J. V. Zanchetta, J. M. Douillard, J. Niezette, and J. Vanderschueren, "A simple general relationship between the dielectric losses measured on divided solids and adsorption thermodynamic," Langmuir, vol. 13, no. 5, pp. 10161019, 1997.

[29] M. M. Dubinin and I. V. Radushkevich, "Equation of the characteristic curve of activated charcoal," Proceedings of the Academy of Sciences of the USSR, Physical Chemistry Section, vol. 55, pp. 331-337, 1947. 
[30] M. G. Kaganer, "A method for the determination of specific surfaces from the adsorption of gases," Proceeding of the USSR Academy of Sciences, vol. 116, pp. 603-605, 1957.

[31] M. M. Dubinin and V. A. Astakhov, "Description of adsorption equilibria of vapors on zeolites over wide ranges of temperature and pressure," in Molecular Sieve Zeolites-II, vol. 102 of Advances in Chemistry, pp. 69-85, American Chemical Society, 1970.

[32] J. P. Hobson and R. A. Armstrong, "A study of physical adsorption at very low pressures using ultrahigh vacuum techniques," Journal of Physical Chemistry, vol. 67, no. 10, pp. 2000-2008, 1963.

[33] J. P. Hobson, "First adsorbed layer of Helium at $4.2^{\circ} \mathrm{K}$," Canadian Journal of Physics, vol. 37, pp. 300-312, 1959.

[34] N. Endow and R. A. Pasternak, "Physisorption of Xe and $\mathrm{Kr}$ on glass and molybdenum," Journal of Vacuum Science \& Technology, vol. 3, pp. 196-201, 1966.

[35] D. M. Young and A. D. Crowel, Physical Adsorption of Gases, Butterworths and Co, London, UK, 1962.

[36] J. M. Cases, I. Bérend, G. Besson et al., "Mechanism of adsorption and desorption of water vapor by homoionic montmorillonite. 1. The sodium-exchanged form," Langmuir, vol. 8, no. 11, pp. 2730-2739, 1992.

[37] I. Bérend, J.-M. Cases, M. François et al., "Mechanism of adsorption and desorption of water vapor by homoionic montmorillonites: 2. The $\mathrm{Li}^{+}, \mathrm{Na}^{+}, \mathrm{K}^{+}, \mathrm{Rb}^{+}$and the $\mathrm{Cs}^{+}$exchanged form," Clays and Clay Minerals, vol. 43, no. 3, pp. 324-336, 1995.

[38] J. M. Cases, M. François, J. P. Uriot, L. J. Michot, I. Bérend, and F. Thomas, "Mechanism of adsorption and desorption of water vapor by homoionic montmorillonite: 3 . The $\mathrm{Mg}^{2+}, \mathrm{Ca}^{2+}, \mathrm{Sr}^{2+}$ and $\mathrm{Ba}^{2+}$ exchanged forms," Clays and Clay Minerals, vol. 45, no. 1, pp. 8-22, 1997.

[39] G. Halsey, "Physical adsorption on non-uniform surfaces," The Journal of Chemical Physics, vol. 16, no. 10, pp. 931-937, 1948.

[40] T. L. Hill, "Theory of physical adsorption," Advances in Catalysis, vol. 4, pp. 211-258, 1952.

[41] J. Frenkel, Kinetic Theory of Liquids, Clarendon Press, Oxford, UK, 1946.

[42] J. D. Russel, V. C. Farmer, and B. Velde, "Replacement of $\mathrm{OH}$ by OD in layer silicates, and identification of the vibrations of these groups in infra-red spectra," Mineralogical Magazine, vol. 37, no. 292, pp. 869-879, 1970.

[43] J. Mamy, Recherches sur l'hydratation de la Montmorillonite: propriétés diélectriques et structure du film d'eau [Ph.D. thesis], Paris, France, 1968.

[44] G. J. Rosasco and J. J. Blaha, "Raman microprobe spectra and vibrational mode assignments of talc," Applied Spectroscopy, vol. 34, no. 2, pp. 140-144, 1980. 

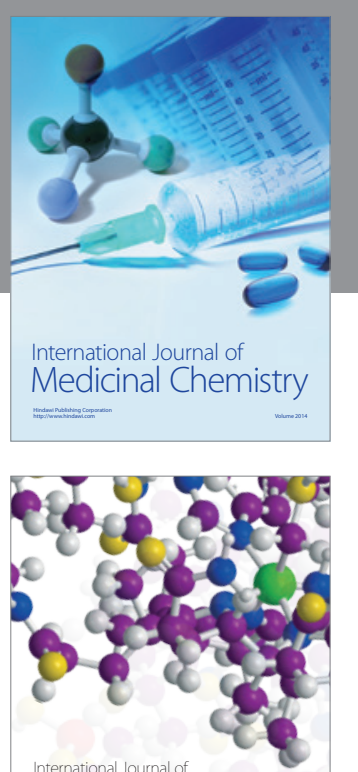

Carbohydrate Chemistry

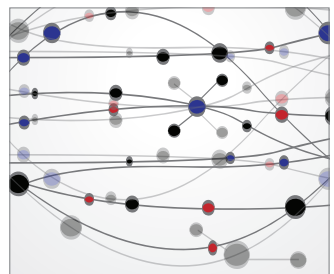

The Scientific World Journal
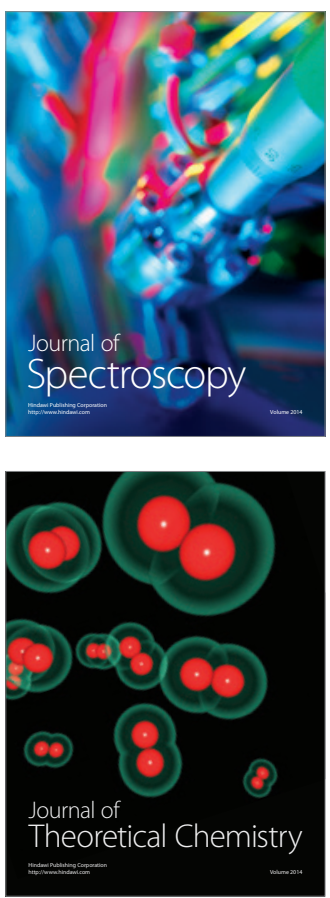
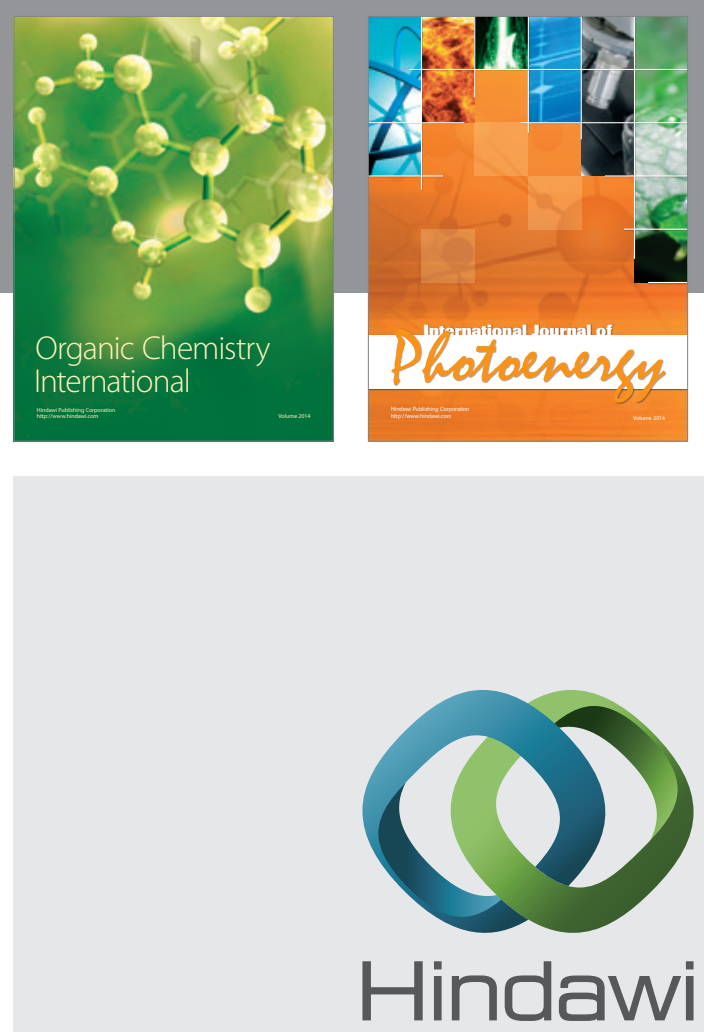

Submit your manuscripts at

http://www.hindawi.com

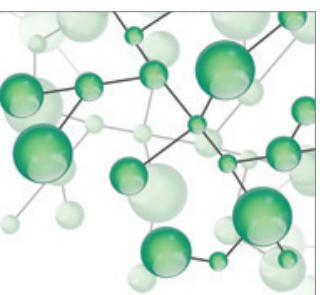

International Journal of

Inorganic Chemistry

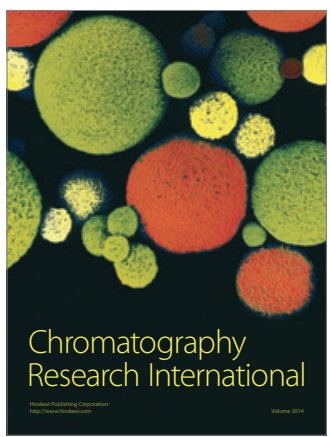

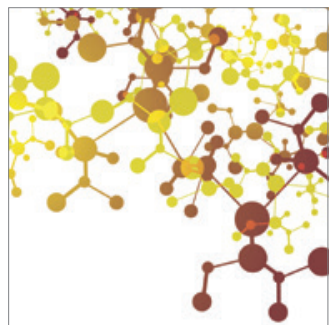

Applied Chemistry
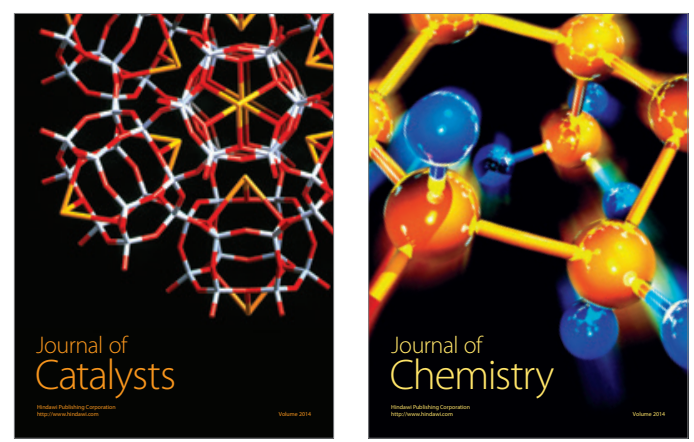
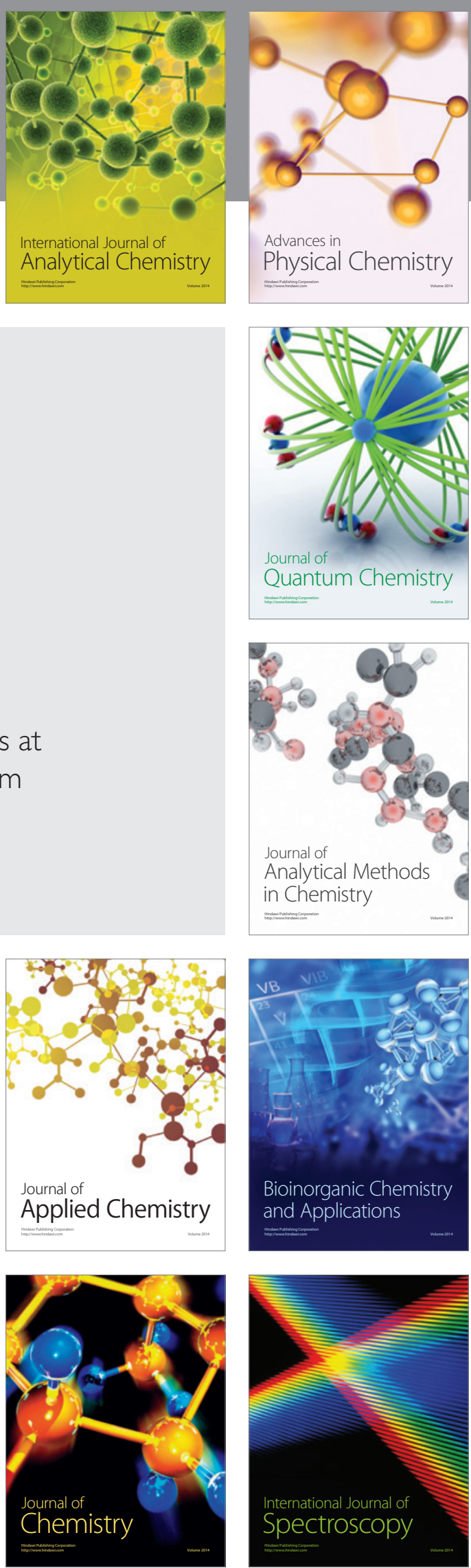